\title{
NEAR FIELD EFFECTS OF SMALL SCALE WATER BLEVE
}

\author{
ROLAND EYSSETTE ${ }^{2}$, FREDERIC HEYMES ${ }^{1}$, JAMES CRAWFORD ${ }^{2} \&$ ALBRECHT M. BIRK $^{2}$ \\ ${ }^{1}$ Ecole des Mines d'Alès, LGEI/ISR, France \\ ${ }^{2}$ Department of Mechanical and Material Engineering, Queen's University, USA
}

\begin{abstract}
This paper presents preliminary results collected with a new experimental apparatus developed to collect close-in overpressure data from small scale water BLEVE. The apparatus consists of a sealed aluminium tube pressurized and heated up until rupture, representing a realistic pressure vessel failure. Various types of failure were obtained, from partial opening to catastrophic failure, giving detailed data on the blast overpressure, the load generated by the failure on the ground, and the transient pressure in the vessel. High speed imaging of the failure gives new insight to the close-in conditions of the explosive release. A CFD modelling work aimed to investigate the ability of CFD to model the blast pressure peaks from a pressurized vapour space. A shock tube configuration was selected, and a series of experiments were performed to provide experimental data. A very good agreement was observed which validates a first step in BLEVE simulation by CFD.
\end{abstract}

Keywords: water BLEVE, near field data, overpressure, superheated, CFD.

\section{INTRODUCTION}

Pressurized vessels are commonly used in the industry to store PLG (Pressure Liquefied Gas). They are part of the industrial landscape, storing various type of PLG, carbon dioxide, propane, ammonia, etc. The failure of these pressurized vessels leads to fast expansion of the gas from high pressure (2-5 MPa) to atmospheric pressure, and sometimes a BLEVE occurs. This powerful type of explosion can be devastating in terms of industrial and material damage, but also in terms of human life. Many accidents have happened through the years and yet the understanding of this accident is still not complete. Moreover, the power of this kind of explosion could be used as a terrorist weapon on civil roadway infrastructures or industrial sites and buildings. The need to mitigate this accident requires a deeper understanding on the triggering process and initial failure.

\subsection{BLEVE definition}

Reid [1], defined a boiling liquid expanding vapour explosion (BLEVE) as an explosion due to the superheated liquid state of a Pressure Liquefied Gas (PLG). When a vessel holding a PLG near its superheat limit fails, the sudden pressure drop results in the explosive boiling of the liquid due to homogeneous nucleation. Homogeneous Nucleation requires a high degree of superheat and this requires storage with no surface roughness and no suspended particles for boiling nucleation sites. This is very unlikely in practical storage systems.

A common case of failure of pressure vessels is due to fire engulfment. The fire first heats up the liquid phase, increasing both pressure and temperature inside the vessel. Simultaneously, the upper part of the tank wall, in contact with the vapour phase, heats up dramatically due to a poor heat exchange with the vapour phase, unlike the liquid phase. The high temperature weakens the tank wall until it can no longer contain the high pressure. Plastic deformation in the vapour space wall due to hoop stress leads to the formation of a fracture opening. This opening releases the high-pressure vapour in the vessel and this leads to boiling in the liquid. At the same time, the crack will propagate or not, depending on the length of the weakened area of the vessel and the pressure exerted by the released fluid. If 
the crack propagates to the total length of the vessel, it will open fully to release the BLEVE. The structure is then destroyed, flattened on the ground, leading to an explosion due to the sudden release of the vapour and liquid.

Inside the vessel, when it opens, a chain of release mechanisms happens, with varying timing depending on the speed of the opening. First the pressurized vapour phase expands in the surrounding, with expansion waves travelling inside the vessel, bringing the liquid to a state of superheat. Depending on the degree of superheat reached and nucleation sites present in the vessel, the liquid will start to flash, or boil explosively. This boiling induces swelling, vapour generation and expansion.

\subsection{Close-in overpressure hazard}

A BLEVE induces various hazards: fireballs, flash fires or possibly vapour cloud explosion with flammable PLG, projection of vessel fragments, and blast overpressure. This work is focused on close in blast and overpressure from a BLEVE. This information is needed by emergency responders and critical infrastructure managers. There is very little data available on close in BLEVE blast hazards.

Measurements have been done on such phenomenon by Giesbrecht et al. [2], Birk et al. [3] and others. Most of the data applies to the far field overpressure. However, the near field is source of interest too, as it has a direct impact on surrounding infrastructure, such as tunnels, bridges, buildings. The strength of this close in field requires the understanding of each phase contribution in the build-up of shock waves.

Laboureur [4], did some experiments with supercritical fluid explosions, capturing interesting patterns of shocks, condensation clouds and two-phase flows that expand out of the tube. Chen et al. [5], focused on the explosive boiling process, capturing high speed images of the boiling and relating them to overpressure measurement in his apparatus. Van den Berg [6], built a numerical model assuming that the vessel was failing and the liquid was flashing instantaneously, characterizing that as the worst-case scenario in terms of energy release and shock formation. His blast charts give an interesting data for comparison to close in blast measurements. However, the authors stay critical on the idea of worst case scenario, and wonder if the shape of opening, the directionality of the propagation and the timing of the combined phenomena couldn't make this case worse.

The consequences of the blast hazards are strongly related to its close-in mechanism. Moreover, the closer to the vessel, the stronger the overpressure. This brings the need of understanding the physics of blast release at the local scale, near the failure zone.

The blast is generated by the sudden drop in pressure from the vessel opening to the ambient surrounding, which brings the vapor inside the vessel to expand suddenly. The resultant pressure waves catch up to each other and accumulate generating a shock wave. Various parameters will influence the strength of the overpressure, as well as the shape the shock wave:

- Shape of the opening: fully open vs partial failure.

- Vessel pressure at rupture.

- Temperature distribution in the liquid and vapor phase.

The two last parameters characterize how much energy is stored in the system before explosion. The TNT-energy model for propane can be used to gage the explosive power in equivalent mass of TNT. This model showed interesting results for far field, but the behaviour of the explosive and the vessel are mismatching for close-in results. To have a better 
description of the near field, the contribution of the different phases in the explosion need to be understood. Two coupled mechanisms are generating overpressure at failure: the vapor phase expansion and the liquid boiling. The global aim of this work is to model BLEVE pressure effects by CFD. In this paper, the focus is done on pressure peaks and CFD modelling of the vapour phase.

In the following, we will present the experimental apparatus set up to make some near-field overpressure measurements, and show some preliminary results with an initial analysis relevant to the associated literature.

\section{EXPERIMENTAL APPARATUS}

The apparatus consisted of a 50-mm diameter tube, sealed and partially filled with water. Burners on top and bottom of the cylinder were available to heat up the fluid inside to increase the pressure and to weaken the top of the tube to generate the failure.

Low speed instrumentation was available to get information on pressure and temperature of each phase inside the vessel before failure. High speed instrumentation was set (blast gages 3 and 4 , load cells 5 and 6 , high speed pressure transducer 7) to measure data when the failure happened, such as blast overpressure on top of the tube with different gage orientations, load generated by the explosion on the ground, and transient pressure inside the vessel while expansion and explosive boiling took place.

The experimental protocol started with filling up the tube with water at atmospheric pressure, with $60 \%$ volume fraction of liquid. Then, the tube was sealed and heated from the bottom to a chosen preheat state. The air was vented from the tube during this heating. Finally, the top burner was ignited to weaken the top of the tube until failure. In some tests, a valve was opened to vent vapour to simulate a pressure release valve to regulate the failing pressure.

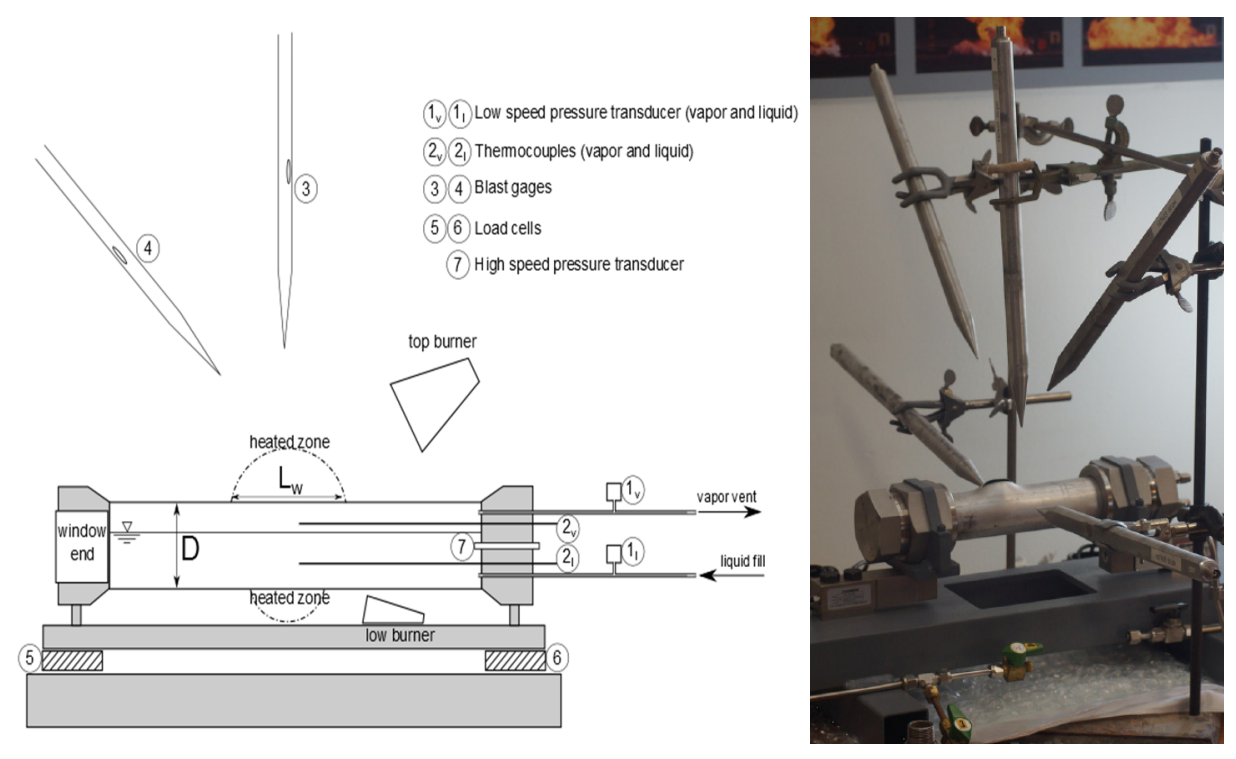

Figure 1: Small scale blast experimental apparatus. 


\section{RESULTS AND DISCUSSION}

The first experimental campaign involved 20 tests, covering a range of typical failure modes, and led to some interesting preliminary results. The main objective of this experimental campaign was to get some blast data out of this new apparatus, from different failing mode (partial and catastrophic failure). So far the tests were only done with a filling of $60 \%$, to evaluate the capability of the apparatus to generate BLEVE. The main varying parameters in the tests were:

- Fire conditions: to obtain different failure, the tube was heated with different burners and burner positions.

- $\quad$ Failing pressure (with Pressure Release Valve simulation for some tests).

A total of 20 cylinders have been used, with a total of 5 calibration tests, 6 partial failures and 9 BLEVEs

Fig. 2 represents a PT-diagram of water, with the PT coordinate of the failure of each test. It is noticed that BLEVE happen at higher pressure than partial failure but still along the saturation curve (the 3 lower BLEVE points are tests with thin wall). It shows the superheated characteristic of the fluid before failure and the higher energy release of BLEVE.

Another way to map the tests is to represent the crack length to the failing pressure (Fig. 3 and Fig. 4). Interesting trends appear in such a graph. First, all tests with a rupture ratio L/D larger than 1 are BLEVEs. Second, a clear separation appears between BLEVE failing pressure and partial failure pressure (obvious for thick wall, less for thin walls). Refining this mapping could lead to an estimation of a critical failure pressure, transition between partial and catastrophic failure.

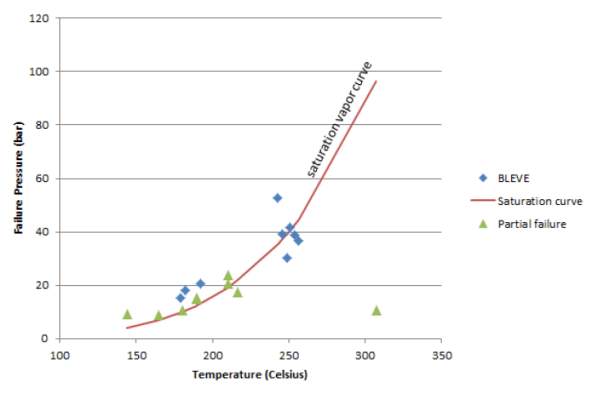

Figure 2: PT-diagram of water tests.
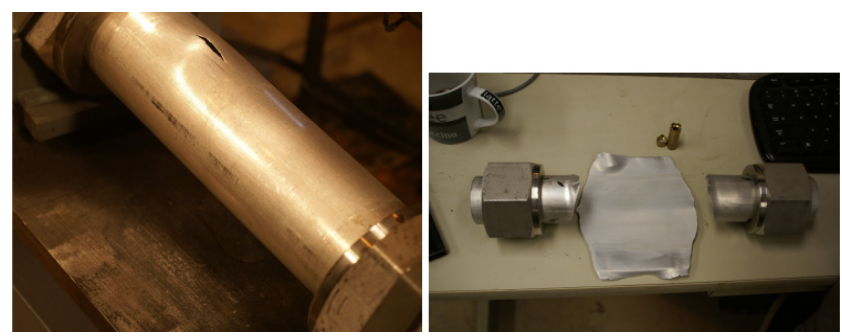

Figure 3: Examples of the 2 failure type obtained (Left: thin wall tube Pfailure $=15.4$ bar; Right: thick wall tube Pfailure $=28.8$ bar). 


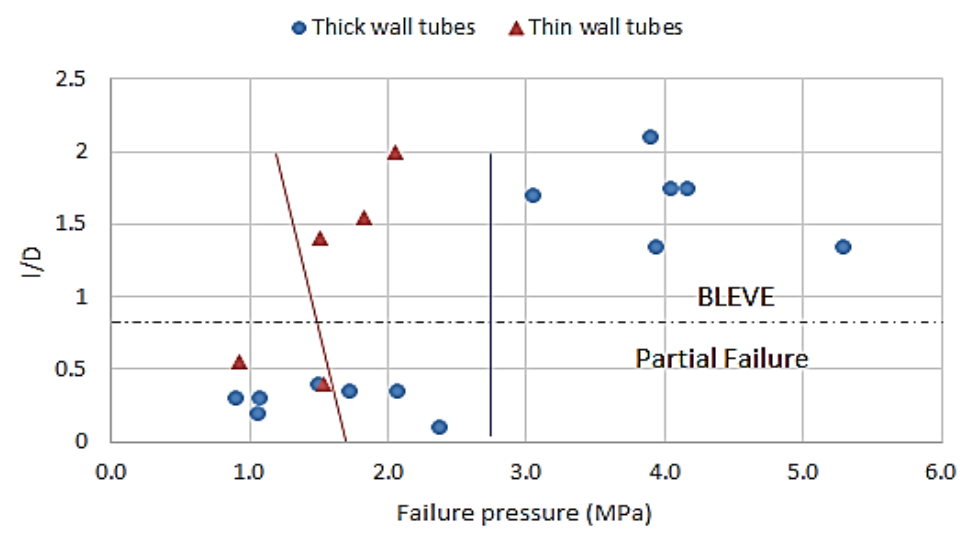

Figure 4: Failure length and pressure as parameters for failure modes.

Those tests brought some interesting data concerning blast overpressure (Fig. 5), load generated by the explosion (Fig. 6), pressure inside the vessel at explosion (Fig. 7). Some timing analysis, as well as comparison with literature will be done to add this new set of data to the research domain.

\subsection{Failure type mapping}

The apparatus was designed to obtain partial and catastrophic failures. Both were obtained by varying fire conditions and pressure in the vessel at failure. Both thick $(1.8 \mathrm{~mm})$ and thin $(0.9 \mathrm{~mm})$ walled tubes were tested. The length of the failure on the opened vessels was measured, scaled by the diameter of the tube and mapped with failure pressure (Fig. 4). A clear separation between BLEVEs and partial failures was observed on both axes. For the failure length, it appears that $\mathrm{L} / \mathrm{D}=1$ is a threshold length between the two failure mode. In terms of pressure, a critical failing pressure appears for thick wall tube between 2.5 and $3 \mathrm{MPa}$. It is less clear for thin wall tubes, probably because of a higher dependence on the heating conditions. However $\mathrm{Pf}=1.5 \mathrm{MPa}$ shows a critical jump from partial to total failure. More tests are required to refine the characterisation of this critical pressure, and the influence of fire conditions.

\subsection{Blast data}

Catastrophic failures gave interesting blast overpressure traces. Fig. 5 represents the data from a test with blast gages on top of the tube and at 45-degree angle around the diameter of the tube, at the same distance from the opening of the tube. The pressure trace of the vertical gage shows a first peak (A) followed by a sharp drop (B), characteristic of a blast wave. Then a second peak $(\mathrm{C})$ follows shortly the previous drop. This could be interpreted as the vapour expansion cloud hitting the gage. After a small rest (D), a last peak of pressure (E) is detected, and again a drop in negative gage pressure (F). It seems to reveal different shock or strong pressure waves building up with delay. What is unknown is which phase of the fluid generates these overpressures. 
Comparing the two gauges shows the 45-degree angled gage signal is about half the strength of the vertical one. This gives an indication of the directionality of the overpressure development, due to the shape of the opening of the vessel.

\subsection{Load data}

To understand the impact of BLEVE on civil infrastructure such as bridges, the force and impulse of the explosion on the ground was measured (Fig. 6). No such data has been found in the literature for comparison so far. A quick estimation of the load is made by calculating the load from the surface area of the tube $(\mathrm{A}=\pi \times \mathrm{D} \times \mathrm{L})$ and the failure pressure $(\mathrm{F}=\mathrm{A} * \mathrm{Pf})$, giving an estimated load of $33 \mathrm{kN}$. Data are giving a maximum load of $28 \mathrm{kN}(14 \mathrm{kN}$ per load cell). The calculation is an overestimation because it doesn't consider the rupture pressure transient. However, it validates the order of magnitude of the load measured.

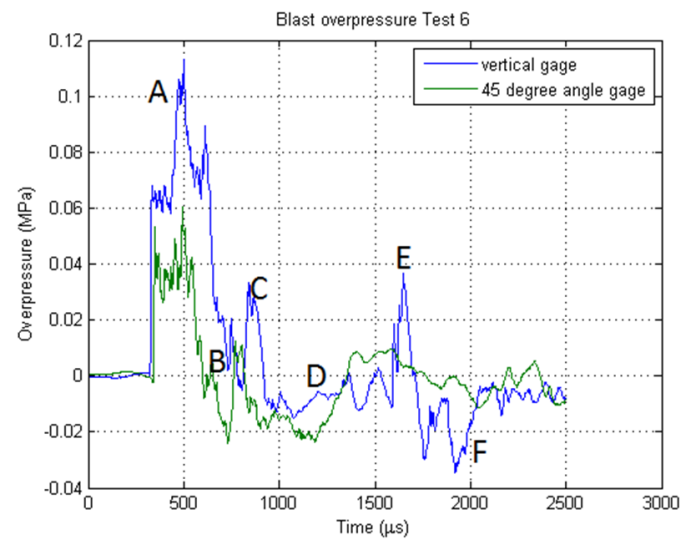

Figure 5: Blast measurements.

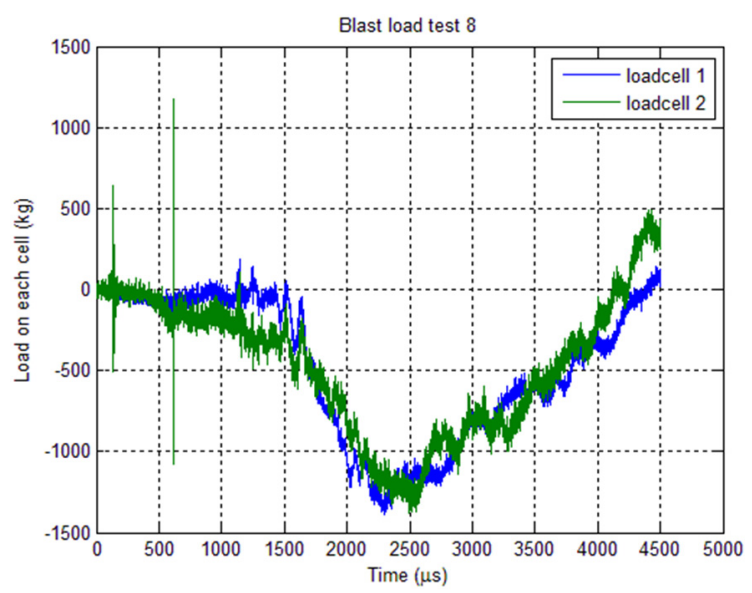

Figure 6: Load data from a water BLEVE failure. 


\subsection{Transient pressure}

Transient pressure data gives us information on the evolution of the boiling process inside the tube. The present study uses one pressure transducer, initially in the liquid phase. The first drop observed on Fig. 5 most probably shows the expansion wave going through the liquid phase, bringing it to superheated state. Then the boiling happens and brings the pressure up. It can be noticed also that the pressure rises higher than its initial value before total failure of the tube. This reveals the power of the explosive boiling phenomenon. Additional testing is required before significant conclusions are drawn to confirm that the signal measured is a good representation of the pressure in the tube, as the tube end was ejected at a high rate of speed from the base plate when the explosion happened.

\subsection{High speed imaging}

High speed imaging was taken from the water BLEVE tests. These images (Fig. 8) show the process of opening of the vessel. A crack appears, releasing a first jet of expanding vapour. The crack propagates longitudinally to the extremities of the tube. When the crack reaches the ends of the tube, it keeps on propagating along the tube diameter until it separates a piece of tube. At the same time, the sides of the tube flatten on the base plate, due to the pressure forces of the fluid.

This sequence of pictures shows also an interesting feature in the behaviour of the expanding fluid. The first 6 pictures of Fig. 8 show a jet and a first condensation cloud, due to the vapour phase expansion. From Fig. 7, a 2nd cloud emerging from the vessel can be distinguished, and really visible in the last pictures. It can be associated to the explosive boiling process, delayed from the first expansion. The sharp spikes of this 2 nd cloud reveal the violence of the boiling phenomenon that is not seen on the 1st cloud.

\section{CFD MODELLING}

In order to understand the pressure peaks (Fig. 5) a CFD modelling was undertaken. The aim was to model the shock wave created by the pressurized vapour space. This was performed by considering a shock tube device. A classic case to test a solver in compressible flow conditions is the shock tube simulation. The presence of a moving shock wave and expansion waves allow to test 2 main components of compressible flows: a moving discontinuity and a varying smooth part.

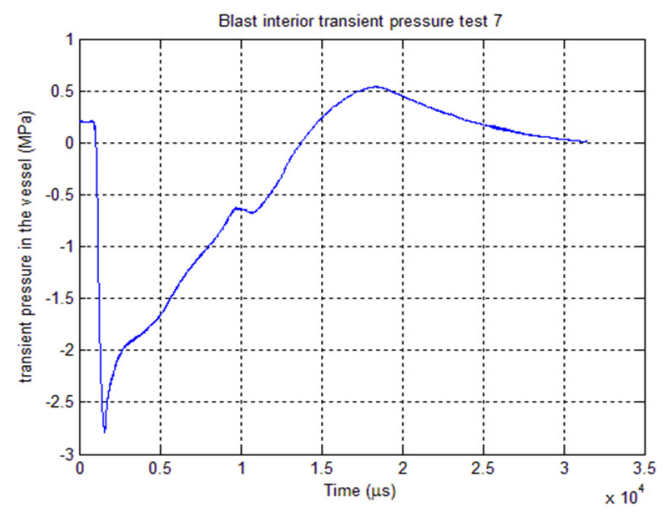

Figure 7: Transient pressure in the tube. 
The difference between pressure-based and density based solvers for shock tube case was investigated. Using 3 different meshes (1D, 2D, 2D-axis symmetric), the conclusion is that a density-based solver with explicit time stepping describes the best shock without wiggles, with dissipation acceptable in the expansion wave part.
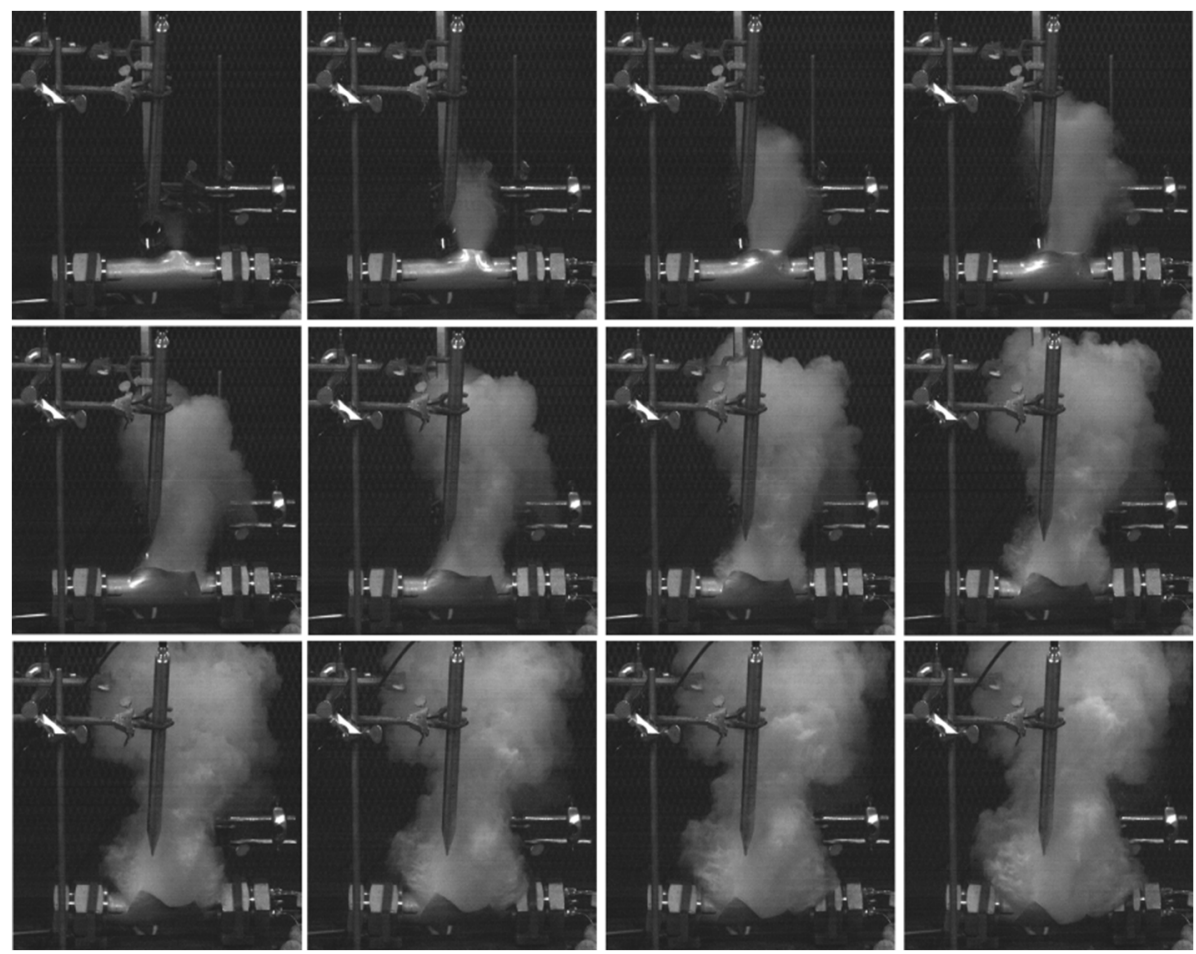

Figure 8: High speed images of a catastrophic vessel failure.
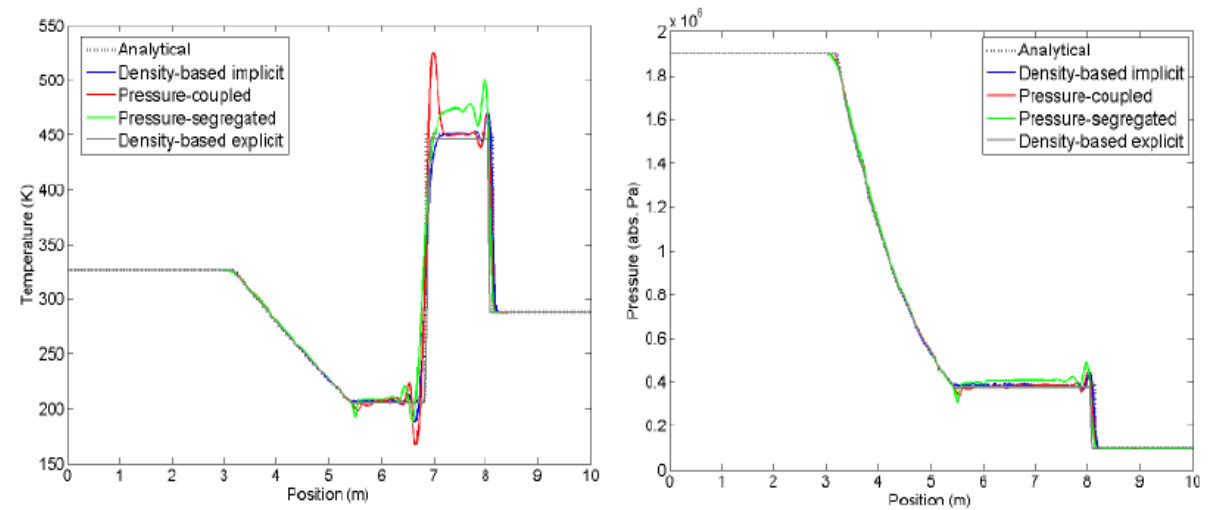

Figure 9: Temperature and pressure profiles of a shock tube. 
Table 1: Comparison of overpressure between CFD results and experimental data.

\begin{tabular}{|l|l|l|l|l|}
\hline Input & Test 1 & Test 2 & Test 3 & CFD \\
\hline P1 $(\mathrm{kPa})$ & 101.325 & 101.325 & 101.325 & 101.325 \\
\hline P4 $(\mathrm{kPa})$ & 394 & 399 & 394 & 398 \\
\hline T1 $(\mathrm{K})$ & 295 & 295 & 295 & 295 \\
\hline T2 $(\mathrm{K})$ & 310.8 & 311.4 & 311.3 & 311.0 \\
\hline $\mathrm{dP}(\mathrm{kPa})$ & 95.3 & 91.5 & 94.4 & 94.1 \\
\hline
\end{tabular}

Fig. 9 demonstrates the ability of CFD to reproduce accurately the pressure from a shock tube. The results show a good match for overpressure values between CFD and experiments (Table 1). The conditions have been matched at their closest in this purpose (tube geometry, initial conditions). This validates the values given for the plateaus of pressure and temperature with Pressure-Based Coupled solver. In further simulations, the model geometry will be adapted to other experimental requirements for sake of comparison.

The next step will be to model the blast from vapour space by a realistic cylindrical configuration and a dynamic opening.

\section{CONCLUSION}

This small scale BLEVE blast apparatus allows cost effective experiments to be performed quite quickly. The results obtained in terms of pressure measurements show interesting features when compared to the literature. Other results also add some novel data, for example the ground load generated by such and explosion, but also the high-speed imaging of the opening process of a BLEVE.

Next, a deeper analysis is required to treat the data obtained. Analysis will be conducted to look at shock velocity calculation, overpressure decay and directionality quantification. Impulse of the load data needs to be extracted to understand its potential impact. Transient pressure will be compared in more detail with literature. Moreover, comparison of the timing of the different events may be required to understand the contribution of each phenomenon in the pressure generation. Experiments will be improved with more repeatable fire conditions, such that its influence can be better understood, as well as better optical techniques including high speed shadowgraph. The purpose of this is to be able to visualize shockwaves propagating after failure. Further CFD work will be undertaken in order to simulate the blast due to the vapour space energy.

\section{REFERENCES}

[1] Reid, R.C., Possible mechanism for pressurized-liquid tank explosions or BLEVE's. Science, 203(1979), pp. 1263-1265.

[2] Giesbrecht, H., Hess, K., Leuckel, W. \& Maurer, B., German Chemical Engineering 4, pp. 305-314, 1989.

[3] Birk, A.M., Davison, C. \& Cunningham, M., Blast overpressures from medium scale BLEVE tests. Journal of Loss Prevention in the Process Industries, 20, pp. 194-206, 2007.

[4] Laboureur, D., Experimental Characterization and Modelling of hazards: BLEVE and BOILOVER, PhD thesis, Université Libre de Bruxelles, 2012.

[5] Chen, S., Sun, J. \& Wan, W., Boiling liquid expanding vapor explosion: Experimental research in the evolution of the two-phase flow and over-pressure, Journal of Hazardous Materials, 156, pp. 530-537.

[6] Van den Berg, A.C., American Institute of Chemical Engineering Process Safety Progress 27, pp. 219-224, 2008. 